\title{
Computational structural mechanics
}

\section{Foreword}

Advances in computer science and technology have had a profound influence on structural engineering. A new discipline called computational structural mechanics (CSM) has emerged and a huge software industry has grown along with it. CSM has virtually developed out of a technique called the finite element method (FEM). Powerful general purpose FEM packages in the Computer-Aided-Design/Computer-Aided-Manufacturing cycle automate the use of structural analysis techniques to check designs quickly for safety, integrity, reliability and economy. Very large structural calculations can be performed to account for complex geometry, loading history and material behaviour. Such calculations are now routinely performed in aerospace, automotive, civil and mechanical engineering, oil and nuclear industries.

This special issue is dedicated to recent developments in finite element methodology leading to the design, validation and use of sophisticated software algorithms to generate structural information, and to assemble and solve them, maintaining careful book-keeping of all data throughout, and subsequently to process the results in a manner helpful to the designer for decision making.

Most finite elements in use today in general purpose packages are based on the minimum total potential principle (displacement elements). In recent years, multifield variational principles have been seen to provide a broader conceptual framework to interpret the method. The first paper "Finite element analysis and the stress correspondence paradigm" deals with the paradigm change that follows when the $\mathrm{Hu}$-Washizu basis is used instead of the minimum total potential basis for the interpretation of how the finite element method works.

Considerable progress has taken place in developing computational techniques for nonlinear static and postbuckling problems and its application to damage assessment in composite structures. The second paper by B P Naganarayana and S N Atluri on computational modelling and analysis of interactive buckling and delamination growth in composite structures reviews this subject.

An important design driver in aerospace applications is the behaviour of structural parts under cyclic loading. The third paper by B Dattaguru, on the role of elasto-plastic analysis under cyclic loading in fatigue crack growth analysis, shows how this is performed. The fourth paper, by Dipak K Maiti and P K Sinha, on "Low velocity impact analysis of composite sandwich shells using higher-order shear deformation theories" addresses another topic of design interest. 
The modelling of complex structures using pre-processing and high resolution, high throughput graphic devices and integration of analysis programs into CAD/CAM systems requires automated mesh generation and subsequent regeneration - how this can be done adaptively is discussed by C S Krishnamoorthy and S Mukherjee in their paper titled "Adaptive finite element analysis with quadrilateral elements using a new $h$-refinement strategy."

Although India has made useful contributions in areas related to element technology, i.e. the design of mechanics algorithms for a wide range of structural and thermomechanical problems, not much has been done in the way of integration of these mechanics algorithms and solution capabilities with pre- and post-processing packages into general purpose packages. These large packages are now commonplace in a wide spectrum of engineering activity in the country but, sadly, most of this software is acquired from abroad. There has not been a serious commitment to the design and maintenance of a package from within the country. A medium-sized package for structural analysis, named FEPACS, for analysis of composite structures is being developed by the National Aerospace Laboratories at Bangalore. The last paper by B P Naganarayana, Gangan Prathap and B R Somashekar, titled "FEPACS: A computational tool for linear structural analysis" shows how the Cconcepts are used as the basis for developing a library of elements and discusses the steps taken to enhance the general purpose capabilities of the FEPACS package. 